\title{
ARTÍCULOS
}

\section{CARTOGRAFÍAS POSTESTRUCTURALES ENTRE LAS PRÁCTICAS ALTERNATIVAS EN SALUD Y LAS NUEVAS ESPIRITUALIDADES}

\author{
POSTSTRUCTURAL CARTOGRAPHIES ON THE BORDERS BETWEEN HEALTH \\ ALTERNATIVE PRACTICES AND NEW SPIRITUALTIES
}

\author{
José Palacios Ramírez ${ }^{1}$ \\ Universidad Católica San Antonio
}

Recibido: 22 de noviembre de 2017; Aprobado: 3 de julio de 2018. Publicado online: 15 de marzo de 2019

Cómo citar este artículo / Citation: Pa lacios Ramírez, José. 2019 . «Cartografías postestructurales entre las prácticas alternativas en salud y las nuevas espiritualidades». Disparidades 74(1): e008. doi: <https://doi.org/10.3989/dra.2019.01.008>.

RESUMEN: La articulación de viejas y reinventadas espiritualidades con prácticas alternativas ligadas a la salud, constituye una pieza clave para abordar las transformaciones culturales en las formas de vida actuales. Este trabajo es esencialmente una propuesta de reflexión teórica, aunque se apoya en la presentación de algunos aspectos incidentales extraídos de procesos etnográficos desarrollados durante los últimos años, que muestran claramente el carácter ubicuo, difuso y elusivo de estas prácticas, especialmente cuando se trata de elaborar miradas explicativas de conjunto. La idea central de estas reflexiones será básicamente: ¿qué podemos aprender de esta fragmentariedad?, ¿qué ocurre con los aspectos de estas nuevas articulaciones diseminados en otros tipos de prácticas? Con base en esto, trataremos de delimitar los principales perfiles y problemáticas que ofrece este complejo e interesante objeto antropológico de reflexión, apoyándonos en propuestas conceptuales posestructuralistas.

PALABRAS CLAVE: Subjetividades; Nuevas espiritualidades; Prácticas alternativas en salud; Realidades emergentes; Ambiente holístico.

\begin{abstract}
The interaction between old and reinvented spiritualties and alternative practices related to health is a key element in addressing cultural transformations in current ways of life. Though based on the presentation of a number of incidental aspects, extracted from ethnographic processes developed over recent years, this work essentially offers an approach for theoretical reflection, showing the ubiquitous, vague and elusive nature of such practices, especially when seeking to compile a set of explanatory viewpoints. The key questions addressed include: What can we learn from this fragmentation? And what is happening with new aspects of these interactions found in other kinds of practice? In the process, we will seek to demarcate the main outlines and issues raised by this complex and interesting anthropological issue, basing our reflections on poststructuralist conceptual proposals.
\end{abstract}

KEYWORDS: Subjectivity; New Spiritualties; Alternative Health Practices; Emergent Realities; Holistic Milieu.

Copyright: (C) 2019 CSIC. Este es un artículo de acceso abierto distribuido bajo los términos de la licencia de uso y distribución Creative Commons Reconocimiento 4.0 Internacional (CC BY 4.0).

1 Correo electrónico: jpalacios@ucam.edu. ORCID iD: <https://orcid.org/0000-0001-8607-6425>. 


\section{DE PROBLEMAS Y ENFOQUES}

Posiblemente la articulación de viejas y reinventadas espiritualidades con prácticas alternativas ligadas a la salud, constituye una de las líneas maestras para abordar las transformaciones culturales en las formas de vida actuales ${ }^{2}$. Esto se debe no solo a las características específicas de los conjuntos de ideas, prácticas o formas de sociabilidad que podrían incluirse en dicha etiqueta teórica, sino también, y posiblemente sobre todo, a una serie de procesos que actúan como marco de condiciones políticas y económicas y que confluyen y se interconectan atravesando este tipo de prácticas: transformaciones en los procesos de modernización económica, redefinición de los ámbitos público/privado y del margen de acción estatal, emergencia de formas de pluralismo en los sistemas biomédicos, hiperproductivización de las políticas del sujeto, transformaciones en las experiencias y marcos institucionalizados de la espiritualidad, etc. Técnicas orientales de meditación, limpias de malas energías tomadas de otras culturas, cantos armónicos, yoga, ingestiones grupales de ayahuasca o peyote guiadas por chamanes, o formas de adivinación como el I Ching, entre otras muchas, constituyen el tipo de prácticas al que vamos a venir refiriéndonos, si bien nuestra pretensión es dirigir la atención de las reflexiones hacía algunas implicaciones menos llamativas o tenidas en cuenta de manera general al tratar de dar cuenta sobre la extensión de este tipo de fenómenos.

Lógicamente en la caracterización general que supone esta enumeración de procesos se hace referencia esencialmente a las sociedades occidentalizadas, si bien muchos de sus aspectos forman parte de variaciones globales (Collier y Ong 2004). Dicha caracterización apunta al despliegue mundial de formas de organización social vinculadas a lo que de forma general se suele denominar como neoliberalismo. En este caso optamos por apoyarnos en la noción de «liberalismo avanzado» ${ }^{3}$ (Rose 1997: 33

2 En el caso de las ciencias sociales y la antropología española, el desarrollo del fenómeno y también del interés en analizarlo se da con cierto retraso respecto a otros casos. El trabajo de Cornejo y Blázquez (2013) ofrece además de un estupendo marco de reflexión, una revisión bastante sistemática de la producción existente.

3 A saber: a) una nueva relación entre expertos y política donde se daría algo así como un paso de la autonomía a la auditoría, donde la conformación de mercados es una técnica para reorganizar espacios y formaciones sociales; ss.), propuesta por los llamados sociólogos anglofoucaultianos, que hace énfasis en el surgimiento de formas hegemónicas de racionalización social, a partir de la extensión de ciertos repertorios de prácticas. Otro motivo para optar por este planteamiento para nominar el marco de condiciones en el que se desarrollan las prácticas que centran estas reflexiones, es dar cuenta de que lo que hoy denominamos neoliberalismo, articula concepciones y prácticas sociales de muy distinto origen, entre ellas las demandas de autonomía personal, políticas de la identidad o el cuerpo, provenientes de la contracultura y el movimiento new age. Además de esto, nuestro objeto de reflexión presenta un aspecto arquetípico de lo que puede entenderse como el campo de trabajo antropológico contemporáneo, aterriza de distintas formas lo que lan Hacking (2001) Ilamaría "procesos de ajuste», problemáticas ligadas a la dimensión y los efectos sociales de los «avances científico-técnicos», la implementación de formas de intervención social y formas de pensar estos nuevos marcos.

Evidentemente la posición desde la que uno se plantea reflexionar sobre alguna cuestión suele estar muy condicionada por su experiencia. La articulación de prácticas alternativas en salud y nuevas espiritualidades no ha sido un tema de investigación empírica directa para mí. Pero mi interés en el vínculo entre la producción de formas de subjetividad y salud/enfermedad ha hecho que vaya tornándose en un tema que aparecía de manera colateral en distintos trabajos, inspirándome siempre interés. Este trabajo es esencialmente un ejercicio de reflexión teórica, que se apoya en elementos incidentales de experiencias etnográficas recogidas en el transcurso de dos investigaciones durante los últimos años. Las narrativas de miembros de Alcohólicos Anónimos en las que aparecen ideas sobre la conducción apropiada de la conducta y el destino, relacionadas con el bienestar espiritual, prácticas alimentarias y metáforas de purificación energética.

b) una pluralización de las tecnologías sociales, una desgubernamentalización del Estado junto a una desestatalización del gobierno, conectándose estos procesos con una dinámica social que estaría complejizando los actores sociales en juego, nuclearizándolos en sujetos individuales y comunitarios; y c) una nueva especificación del sujeto de gobierno, donde individuos y comunidades son ahora el nuevo medio qué y a través del cual gobernar, lo cual conlleva diferencias en las relaciones de autoridad, un nuevo orden de obligaciones, espacios de acción y decisión. 
O turistas residenciales británicos en edad de jubilación, que deciden trasladar su proyecto de vida al sureste de la península ibérica a partir de argumentos relacionados con el bienestar personal o la búsqueda de la libertad enunciados de un modo muy trascendental. Ambas son manifestaciones parciales, diseminadas e incluso difuminadas, de un fenómeno extensísimo y ubicuo, en las que parece que casi siempre esté de por medio la cuestión, central en nuestros días, de dirimir el bienestar personal, la salud, la autoestima.

Las ideas centrales en torno a la que se articularán estas reflexiones son básicamente: ¿qué podemos aprender de esta fragmentariedad?, ¿qué ocurre con los aspectos de estas nuevas articulaciones que aparecen diseminados en otros campos, tipos de prácticas o ámbitos?, ¿pueden servir para comprender mejor este fenómeno?, ¿qué pueden enseñarnos sobre las lógicas que ordenan todas estas transformaciones socioculturales, que inevitablemente atraviesan las configuraciones actuales del yo, pero que también de algún modo aparecen inscritas en las formas más o menos institucionalizadas de organizar la experiencia de las personas? De manera general, las reflexiones que siguen parten de un marco de ideas y autores relacionados con lo que se suele denominar posestructuralismo. Dichas posiciones ofrecen a mi entender un potencial crítico que puede ser útil para tratar de pensar sobre estas cuestiones desde un ángulo distinto. Siendo algo más explícitos, diríase que dicho potencial crítico se encaminaría en varios sentidos. Primero, esta óptica teórica nos ofrece otra forma de conectar, de poner en relación los aspectos macro de las prácticas que nos ocupan, vinculados a los grandes procesos y marcos de condiciones, y los micro, ligados a las prácticas en sí y sus sentidos. Además, la idea es que la puesta en juego de estas concepciones sobre las relaciones sociales y el poder nos puedan servir para tratar de discernir con una mayor complejidad los juegos entre formas de liberación y sumisión que se dan en este campo de prácticas sociales, ponderando de paso en una medida más ajustada los procesos de creatividad cultural asignados a menudo a este ámbito, toda vez que dicha óptica puede permitirnos también percibir la traslación de determinadas prácticas o principios de estas entre ámbitos sociales distintos en origen.

\section{ALGO MÁS QUE NUEVAS ESPIRITUALIDADES O NUEVOS MOVIMIENTOS EN TORNO A LA SALUD: EL INCIPIENTE RASTRO DE LAS GRANDES TRANSFORMACIONES}

\section{LA RESOCIALIZACIÓN DE LA ESPIRITUALIDAD}

Para abordar las intersecciones entre prácticas alternativas en salud y nuevas espiritualidades, debemos tener en cuenta un conjunto de transformaciones que se han producido durante las últimas décadas en los dos ámbitos, la salud y la espiritualidad. Estos cambios por cierto presentan algunos paralelismos o puntos de conexión: surgimiento de posiciones de contestación y búsqueda de autonomía y des-institucionalización; aumento ostensible del peso específico de la subjetividad y la experiencia individual; pluralización y complejización de las verdades legítimas en dichos campos; así como surgimiento de nuevas respuestas y estrategias por parte de los tradicionales actores legitimados, especialmente la medicina, pero también las grandes religiones.

Un buen punto de partida puede ser denominar de un modo más o menos operativo este cruce de nuevas espiritualidades y prácticas alternativas en salud. A este respecto Cornejo y Blázquez (2013, siguiendo las propuestas de Heelas y Woodhead 2008), apuestan por el término que quizás mejor se aproxima al carácter elusivo de estas realidades, como es la noción de «ambiente holístico»: «(...) nuestra perspectiva del ambiente holístico hacía referencia a una espiritualidad terapéutica y experimental, apoyada en argumentos cientifistas pero crítica con el materialismo de la biomedicina y con el trascendentalismo de las religiones del libro» (Cornejo y Blázquez 2013: 16).

Dicho ambiente holístico, en plena expansión y complejización, tal y como señalan las citadas autoras, vendría a encuadrarse de manera más amplia en el resurgir contemporáneo del llamado movimiento new age y en la rearticulación de una serie de nuevos movimientos sociales, surgidos al calor de las problematizaciones culturales y las experiencias sociales de la llamada contracultura, originados en la década de los sesenta del siglo pasado. En dichos movimientos ha ocupado y ocupa un espacio central una especie de "reconfiguración del vínculo premoderno entre salud, espiritualidad y cuerpo", que desde su praxis supone una crítica a la institucionalización de estos aspectos de la experiencia humana, al tiempo que la muestra de una serie de 
marcos de entendimiento sobre "las formas clasificatorias de la modernidad». Las características comunes del heterogéneo conjunto de prácticas que componen este ambiente holístico (ibid.: 17) serían: «la circulación de todo un lenguaje ligado a la noción de energía; la presencia planteamientos holísticos que pretenden superar los dualismos modernos, especialmente el de cuerpo/mente; la importancia de la experimentación personal, vinculada con la psicología humanista del desarrollo personal», y una sinonimia entre los términos de salud, bienestar y felicidad, que a menudo es calificada de tautológica (Quiceno y Vinaccia 2009: 324).

Este marco precedente conectado con la llamada contracultura se da paralelamente en el caso de las transformaciones en las formas de espiritualidad, sobre todo en la amplificación del interés por otros sistemas de creencias como los orientales, pero igualmente por los de otras tradiciones culturales de distintos períodos. Aunque es necesario tener en cuenta los procesos de secularización y renegociación de las religiones y las religiosidades tradicionales de cada zona particular. En el terreno de la espiritualidad, desde los años setenta viene desplegándose una extensa y heterogénea diversidad de prácticas y sistemas de creencias, en las que la cuestión de la autonomía individual y la libertad ocupan un espacio central. Esto ha hecho que muchos autores hablen de una especie de "mercado" de las creencias o de "espiritualidades a la carta» (De la Torre y Gutiérrez 2005: 53-70), mientras que otros han preferido centrar su atención en las formas creativas de articulación de lo viejo y lo nuevo bajo la idea levistraussiana de bricolage (Hervieu-Leger 2005). En este caso la producción de categorías se encuentra algo más desarrollada que en el caso de las prácticas en salud, si bien dichas categorías tampoco parecen poder aprehender claramente su objeto de interés. Así nociones como "vernacular religion» (Bowman y Valk 2012) o «neopaganismo» (Fedele 2008) parecen interesantes de cara a representar la idea de una religiosidad contemporánea, híbrida y performativa, una religiosidad ligada a la experiencia cotidiana, vehiculada a través de comunidades poco institucionalizadas, al menos en el sentido más ortodoxo, y muy centrada en el individuo. Ahora bien, en definitiva, estas nociones no dejan de presentar los mismos problemas a la hora de ofrecer miradas de conjunto. Sin duda es necesario aún acumular análisis de prácticas concretas y poder realizar comparaciones entre puntos de determinadas continuidades, como puede ser la in- teracción de estos sistemas emergentes con las religiones tradicionales en el Mediterráneo (Fedele y Llera 2011). Resulta relativamente claro que las vías actuales del conocimiento antropológico pasan, de un modo u otro (Rabinow 1988), por un compromiso epistémico con las prácticas como locus etnográfico. Pero esto conlleva también el interés por la búsqueda de claves explicativas de conjunto que aporten perspectiva sobre procesos más amplios, que discurran de fondo, por debajo de los procesos y agentes que quizá presentan aspectos que los hacen más interesantes o más claramente delimitables y por tanto más óptimamente etnografiables. En sentido por supuesto puede resultar muy interesante preguntarnos por el futuro a medio plazo de las grandes religiones institucionalizadas en nuestras sociedades, por los márgenes de redefinición y/o retradicionalización en las coyunturas que posiblemente se disponen a encarar. Pero igualmente supone todo un reto tratar de pensar en el grado de penetración y extensión en grandes sectores de nuestras sociedades, de creencias y representaciones vinculadas de manera más o menos directa con formas alternativas de espiritualidad, que no tienen por qué conllevar la adhesión a un sistema coherente y cerrado de prácticas, pero que sí informan de la presencia extensa y difusa que recoge la metáfora ambiental. Por ejemplo, aun ponderando los aspectos socioeconómicos, con este trasfondo sería interesante preguntarse por la extensión actual de la práctica de la incineración en relación a la más tradicional de la inhumación, o por la extensión del uso de terapias alternativas o complementarias, no tanto desde una óptica cuantitativa sino más bien en un sentido cualitativo.

Las prácticas e ideas encuadradas dentro del ambiente holístico tienen evidentemente historias particulares de adaptación y desarrollo local en distintos contextos sociales, ligados a sus culturas políticas, márgenes de ciudadanía, sociedad civil y participación, pero también en relación a los sistemas sanitarios y asistenciales existentes. Aun así, en general, dichas prácticas parecen asociarse mayoritariamente con las mujeres y con un segmento de clase que, grosso modo y de manera ciertamente problemática, suele denominarse como clases medias. Esto hace que, a la hora de caracterizar los vínculos del llamado ambiente holístico con los movimientos sociales, que en los sesenta marcaron la impronta de estos nuevos posicionamientos, se suelan destacar los aportes del movimiento feminista, especialmente el de los grupos de conciencia (Blázquez y Cornejo 
2013), sobre el papel de otros movimientos como el ecologismo. Algo perfectamente comprensible atendiendo al conocido hecho de que el cuerpo femenino puede considerarse quizá como el objeto privilegiado por la biomedicina en lo que se han denominado procesos de medicalización (Inhorn 2006).

Así el conjunto de prácticas en torno a la maternidad, parto natural, lactancia materna y crianza natural (Blázquez y Montes 2010; Massó Guijarro 2015), sería quizá un ejemplo paradigmático del tipo de patrón difuso sobre el que nos preguntamos aquí respecto al atravesamiento de nuevas espiritualidades y prácticas alternativas en salud. No tanto porque dicho conjunto presente a priori vínculos necesarios con las espiritualidades new age, aunque seguramente sí que pueda aparecer de manera clara en segmentos concretos o en comunidades particulares que quizás no coincidan con los perfiles sociológicos convencionales, sino porque presenta una característica de conjunto aplicable a los sistemas de prácticas alternativas en la espiritualidad y en la salud, quizá también en otros, y ofrece principios, prácticas y relaciones abiertas, modulables en cuanto el grado de compromiso, la duración la cohabitación e incluso la mezcla entre ellos.

De modo que, sin atender a este principio de dispersión/intensificación, resulta complicado calibrar las dinámicas de cambio que subyacen a indicios como que los sectores de la población que mejor encajan con lo que las propias ciencias sociales hemos delimitado como el perfil predominante en este ámbito: mujeres de edad media, de clase media con un cierto capital cultural, que no las salvaguarda de los procesos de desclasamiento, pero que sí obviamente les abre toda una serie de posibilidades, incluyendo el trabajo y consecuentemente la pluralización de una mayor predisposición al autocuidado incorporada en términos de género (Echevarría Pérez 2008). Nos encontramos entonces ante transformaciones de otro tipo de magnitud, que vendrían desarrollándose en y a partir de las formas colectivas de organización de la conciencia, de la subjetividad, por más que como ya se ha mencionado, el cuerpo, la experiencia y las prácticas tengan una importancia fundamental (Scheper-Hughes y Lock 1987; Janzen 2001).

Se asume, pues, la centralidad de ese hilo que conecta la configuración de los grandes marcos socioinstitucionales con los procesos y prácticas que sostienen los individuos de manera particular y/o colectiva en busca de sentido. Otra cosa bien distinta son las formas de cuestionarse por sus implicaciones. Las llamadas teorías de la reflexividad, al poner el foco en cuestiones como la intimidad o la identidad (Beck, Giddens y Lash 1997), asumen algún tipo de superación o evolución de una serie de lógicas sociales anteriores, superadas fruto de las transformaciones tecnológicas (transporte, comunicación, información). Mientras que, por contra, las denominadas visiones posestructuralistas ${ }^{4}$ (Bourdieu 1997; Foucault 2002a), todas en cierto modo conectadas de algún modo con las concepciones maussianas sobre la relación ontológica entre tecnología y persona, tratan, a través del análisis de los procesos de subjetificación o el habitus, de poner el foco en algo subyacente, bien sea la producción de nuevas formas de pensarse en las prácticas como sujetos sociales ${ }^{5}$, o la reproducción de ciertas lógicas en las nuevas formas de definir-operar con los distintos capitales (véase el reciente trabajo de Moreno Pestaña 2016). Por lo que a la postre cuestionan críticamente la naturaleza de las grandes transformaciones socio-tecnológicas, así como también la articulación potencialmente hegemónica de los nuevas discursos y formas de «libertad» y «emancipación del yo» (Ong 2006), en la medida en que sobre ellas se asientan formas emergentes de control social casi en la misma medida que oportunidades también potenciales de constitución de nuevos sentidos culturales de cuestiones tan elementales como la corporalidad, la identidad, el sufrimiento o los vínculos psicosociales.

La extensión de determinadas concepciones o explicaciones sobre los malestares que afectan a las personas en nuestras sociedades, como la necesidad de un reconocimiento y manejo eficiente de las emociones y energías en la vida cotidiana, o la importancia de la búsqueda de una maximización de todas las posibilidades, experiencias y capacidades de cada sujeto, una especie de idea de realización trascendentalizada al ligarse a una definición etérea del bienestar, están tornándose nucleares en nuestras socie-

4 «Foucault enabled us to see different kinds of relations between truth and power, in which power was a matter of the production of truth, and truth was itself a thing of this world, (...) he enabled us to visualize different kinds of relation between practices that sought to know and manage human individuals and the emergence of conceptuations of ourselves as subjects with certain capacities, rights and human nature» (Rabinow y Rose 2003: 3).

5 Puede verse Esposito (2012) con una perspectiva más general; o de forma más concreta el trabajo de Rose (1999: 5-6 y 2003). 
dades en sentidos muy distintos. Asistir a grupos de meditación, a cursos sobre constelaciones familiares, participar en grupos de danza sufí o recibir terapias por imposición de manos, pueden verse como prácticas que sin duda representan reformulaciones de ciertas lógicas metafísicas de largo recorrido en nuestras culturas y órdenes institucionales. Aunque al mismo tiempo ese extenso corolario de prácticas supone también el desarrollo de toda una serie de funciones sociales conectadas con la eficiencia de los sistemas sociales, con la elaboración individual o microgrupal, y siempre segmentada de los malestares diríase estructurales de la vida social actual. Tal vez esto sea más ostensible en las sociedades con un tejido más débil de asistencia social, en Latinoamérica, iglesias protestantes, grupos religiosos de cultos afro y colectivos de orientación espiritual-terapéutica conforman desde su marginalidad todo un entramado complementario que viene a complementarse, o incluso a sustituir, comportándose incluso como actores paraestatales, las funciones de unos espacios de asistencia inexistentes.

\section{LA DESMEDICALIZACIÓN DE LA SOCIEDAD}

Por su parte, en el campo de la salud la centralidad de las percepciones y estados subjetivos de la enfermedad y la salud sería el primero de los dos ejes clave en los procesos de transformación de las últimas décadas (Flores Martos 2011). Más allá de lo que suponen las conocidas declaraciones de Alma Ata u Ottawa, quizá la cuestión fundamental es la focalización de las estrategias políticas en la promoción del autocuidado y la prevención, en la responsabilidad de los sujetos individuales y colectivos sobre sus propios cuerpos sanos. El segundo eje de dichas transformaciones tendría que ver con la pluralización (claro está no necesariamente consensuada o coherente) del conocimiento científico, sobre todo del relacionado con formas de intervención social planificadas. $Y$ aquí se hace necesaria una aclaración: cuando hablamos de una pluralización del conocimiento, de las concepciones y disciplinas científicas sobre la salud, nos referimos como mínimo a dos tendencias distintas, a menudo contrapuestas, pero que coinciden el tiempo. De una parte, una enorme complejización del conocimiento biomédico que, movido por el auge de las "tendencias sociales», se ha rearmado en torno a los descubrimientos en genética o farmacología, intentando conectar a través de la personalización de los tratamientos con los tan mencionados estilos de vida. $Y$ de otra, un enorme auge de las contestaciones y críticas ligadas a la dimensión ético-política de los sistemas médicos.

De hecho, gran parte de los análisis sobre lo que podríamos entender como ambiente holístico han centrado su atención en la relación de las prácticas alternativas con los procesos de medicalización de la vida (Foucault 1992), a menudo desde una percepción algo rígida, que más que alguna conclusión operativa, parece ofrecernos cierta sensación de perplejidad. De forma muy general algunos autores han visto en las terapias alternativas (Baer y David-Floyd 2006) una respuesta de corte humanista a la medicina tecnocrática y oficial. Si bien lógicamente la relación entre terapias alternativas y la biomedicina parece tornarse mucho más compleja, para empezar porque como se ha señalado a menudo, el entramado de prácticas y saberes alternativos parece organizarse a la manera de un mercado, reproduciendo en parte las concepciones individualistas y mercantilistas que desde hace tiempo constituyen parte básica de las críticas a los sistemas biomédicos (Menéndez 1978). Y sobre todo por las formas más o menos implícitas en las que muchos de estos sistemas terapéuticos responsabilizan al individuo de sus padecimientos y estados de salud (Lowenberg y Davis 1994: 584).

El hecho es que esa concepción extremadamente rígida reproduce una idea muy esquemática de la relación entre formas de hegemonía y resistencia, haciendo difícil apreciar la enorme complejización que vienen desenvolviéndose en el campo de la salud. Así, ciertamente se podría hablar de un «enorme ensanchamiento» (Haro 2000: 102) del campo de la salud en nuestras sociedades, y quizá desde esa asunción resulta más adecuado relacionar las terapias alternativas, especialmente las vinculadas a las nuevas espiritualidades, con una forma de «reconquista de los cuidados y los significados de la enfermedad» por parte de las personas, pero aún así la cuestión se nos sigue escapando, al menos parcialmente. $Y$ aquí sería necesario realizar una breve anotación, dicha cuestión se nos escapa por diferentes cuestiones. La primera de ellas porque seguramente el ámbito de las terapias alternativas no ha cumplido las expectativas que se habían proyectado sobre él. Desde los años 90 voces críticas de la antropología médica norteamericana habían visto en estas terapias la oportunidad potencial de llevar a cabo una verdadera «revolución» en salud, que partiera desde los pro- 
pios sistemas asistenciales, desarrollando para ello la potencia de intervención social inscrita desde sus orígenes en los espacios clínicos. Y la segunda porque la extensión e intensificación de esta esfera de actividad social quizá no guarda tanta relación con proveer a las personas de formas de "curación», como con estrategias, discursos y prácticas más relacionadas con dotar a estas de una vida saludable, es decir, con recursos para evitar los riesgos de la enfermedad y el malestar, o también con acompañarlas cuando esto ya ha sucedido. Esto es, precisamente las áreas donde el entramado más puramente biomédico muestra menos capacidad efectiva de intervención. Su actividad se torna muy intensa en áreas muy relacionadas con lo que los antropólogos denominamos el ciclo vital, uno de los espacios clave de medicalización: maternidad y crianza, envejecimiento, acompañamiento en enfermedades terminales.

Cuando se plantea la emergencia de la subjetividad como pieza central en las concepciones sobre la salud y la enfermedad que han ido conformándose durante el siglo $X X$, y que terminan por cristalizar en los pretendidos marcos biopsicosociales actuales, puede parecer lógico pensar que la intensificación de la atención sobre esta, puede relacionarse con la progresiva constitución del ambiente holístico como alternativa ante una biomedicina que sencillamente estaría centrada en ocuparse de los cuerpos. Pero esta relación no está nada clara. Muchos de los autores interesados en los procesos de tecnificación de la medicina actual, tanto en las formas de diagnóstico preventivo, como en los tratamientos, vienen señalando que dichos marcos institucionales altamente tecnificados estarían teniendo un potente efecto en la realidad, precisamente a través de los procesos de constitución de las subjetividades de los pacientes, sus síntomas, narrativas de sufrimiento y experiencias (Del Vechio Good 2007; Biehl y Moran-Thomas 2009), así como en los imaginarios sociales que se están fraguando, altamente impregnados de estas percepciones y experiencias tecnomédicamente mediadas.

$Y$ es que como ya nos recordara en su momento Michel Foucault (1976), en sus reflexiones a propósito de las propuestas críticas de Iván Illich sobre la medicina occidental, la biomedicina, al margen de sus distintas alianzas coyunturales con diferentes ramas de las ciencias experimentales o tecnológicas, siempre tuvo un fuerte énfasis social y por lo tanto político (puede verse también Comelles y Martínez 1993), pues ahí radicaba la magia de la autoridad, la legiti- midad y la autonomía que pretendía ostentar. Resulta complejo apreciar tendencias en la esfera social de la salud y en la biomedicina, toda vez que la salud ya ensamblada como un dispositivo global (Palacios y Rico 2011) apunta procesos distintos, encontrados e incluso contradictorios, que están desarrollándose de manera coincidente en tiempos y espacios. De manera que podemos hablar del énfasis biologicista de la medicina genético-molecular (Rose 2006), al mismo tiempo que de otra tendencia opuesta centrada en la preocupación por la prevención, la cronificación de las enfermedades y los estilos de vida, que incluso parece centrar su atención en la inclusión entre sus herramientas analíticas de aspectos culturales (Comelles 2003; Fassin 2008), aún entendidos de un modo bastante discutible en ocasiones. Igualmente, la acentuación en los procesos de medicalización, vehiculada a través de la profusión de nuevos fármacos y terapias altamente tecnificadas, por ejemplo, en torno al cáncer, parece complementarse perfectamente con la emergencia en las últimas décadas de lo que podría entenderse como procesos de psicologización de la vida (Rose 1998; Vázquez 2005; Han 2014).

\section{FRAGMENTOS ETNOGRÁFICOS INCIDENTALES: RASTREANDO PERFILES DIFUSOS}

Siguiendo con el esquema propuesto al comienzo, en este apartado del texto la idea es presentar unos breves fragmentos etnográficos, aspectos incidentales de sendas investigaciones previas, junto con las curiosidades y, por qué no decirlo, perplejidades que suponen. La idea es que desde su carácter aparentemente anecdótico puedan servirnos en nuestras reflexiones, encaminadas a preguntarnos por otras formas teóricas de observar y por el fenómeno que nos ocupa. Lógicamente cabe aclarar antes de continuar que dichos ejemplos no pretenden fungir como descripciones empíricas que ilustren descubrimientos teóricos, toda vez que como se expresó al comienzo del trabajo este es sencillamente una reflexión teórica. Al contrario, como se viene comentando durante el conjunto del texto, estos breves pasajes relacionados con investigaciones etnográficas centradas en otras temáticas específicas, tienen la intención de mostrar las paradojas, las dudas que este tipo de prácticas sociales arrojan ante cualquier intento de explicación. Precisamente se busca reorientar la atención de la reflexión general sobre lo que supone la articulación, el solapamiento de prácticas alternativas 
ligadas a la espiritualidad o la salud, desde aquellos sistemas, grupos o colectivos minoritarios, que pueden identificarse claramente como poblaciones de estudio que sostienen estas culturas, hacia otros ámbitos mucho más difíciles de delimitar y clasificar, vinculados con la penetración y extensión de este tipo de prácticas y representaciones en la población general, que no milita en este tipo de ámbitos sociales, pero que de manera ecléctica, segmentaria y discontinua hace uso de ellas o sostiene concepciones o creencias ligadas de alguna manera a ellas.

\section{NARRATIVAS EN GRUPOS de AlCoHólicos ANÓNIMOS CONTAMINADAS DEL BIENESTAR NEW AGE}

Casi todo el mundo sabe de manera más o menos aproximada qué son los grupos de Alcohólicos Anónimos (en adelante AA). Fundamentalmente se trata de un programa terapéutico de rehabilitación del alcoholismo que nace a comienzos del siglo XX en Estados Unidos, en un contexto de fuerte problematización moral, médica y jurídica en torno al consumo de alcohol (Valverde 1998). Los grupos AA surgen al calor de agrupaciones religiosas protestantes, alineadas alrededor de la idea de rearme moral de la sociedad norteamericana, pero también bajo la influencia, menos tenida en cuenta, de las comunidades religiosas y las redes de apoyo de la inmigración de distinto origen que por entonces llegaba masivamente al país (Menéndez 2009: 110). Para después, en las siguientes décadas, extenderse de manera extremadamente exitosa por todo el mundo, especialmente en Latinoamérica.

El programa de AA es un programa terapéutico con fuerte énfasis espiritual, que mediante distintas formas de acción simbólica y procedimientos ritualizados (Antze 1987; Cain 1991), articula el proceso de abandono del consumo de alcohol, con la transformación espiritual del individuo, ahora inserto en la red de sociabilidad del grupo (Palacios 2009). En cierto modo puede decirse que AA viene a ser algo así como uno de los primeros prototipos modernos de la articulación de elementos científicos y terapéuticos, estructuras comunitarias no expertas de ayuda mutua y soporte moral, junto con creencias de carácter espiritual. No obstante, es el germen de los actuales grupos de autoayuda. Algo que en parte queda patente cuando se observa la extensión del modelo de los 12 pasos en un sinfín de arenas rela- cionadas con patrones de consumo problemático o de dependencia: comida, sexo, $\operatorname{drogas}^{6} .$.

Además, en el caso de Latinoamérica, la existencia de lo que Eduardo Menéndez (Menéndez y Di Pardo 1982) denominó procesos de alcoholización y del programa AA corre de manera paralela junto al asentamiento de las condiciones de emergencia de lo que hoy denominamos neoliberalismo. Llama la atención la coexistencia en estas sociedades de movimientos como AA y muchas de sus variantes, con lo que constituyen las otras vías de organización comunitaria y atención a los padecimientos psicosociales, como son las comunidades religiosas neocristianas y protestantes o las religiones afro (Giobellina y González 2000; Weidner-Maluf 2005).

Algunas observaciones surgidas en el marco de la realización de una investigación con agrupaciones de AA en la frontera norte de México, me hicieron plantearme hasta qué punto las nociones espirituales de bienestar personal de corte new age, parecen haberse extendido más allá de los límites de dicho fenómeno, es decir, más allá de las comunidades que de manera relativamente estructurada sostienen este tipo de creencias y prácticas. Durante el trabajo de campo, que incluía la realización de entrevistas en profundidad a miembros con cierta antigüedad en el grupo, me encontré con dos sujetos, E. y P., que presentaban perfiles significativamente similares, de hecho, formaban parte del mismo círculo de sociabilidad dentro y fuera de la agrupación. Ambos provenían de estratos socioeconómicos algo superiores a la media del conjunto del grupo, contaban con estudios universitarios y habían sufrido duros procesos de desclasamiento fruto de su problema con el alcohol. En aquel momento ambos desempeñaban lo que podría calificarse como buenos trabajos en puestos administrativos de cualificación media-baja dentro de la administración estatal, si bien es cierto que estos respondían a un nivel de cualificación y reconocimiento menor que el que ellos ostentaban.

6 Recientemente, a través de un alumno de posgrado que está realizando una investigación sobre comunidades terapéuticas en Michoacán, México, he tenido noticia de la aparición de alguna experiencia muy curiosa en este sentido. Se trata de agrupaciones al estilo de AA que están abriendo sus puertas, y realizando juntas terapéuticas, pero $\sin$ la necesidad de que los participantes presenten ningún tipo de conducta adictiva, simplemente con el objetivo de que las personas puedan expresar y trabajar sus malestares vitales. 
Tanto P. como E. eran asiduos lectores de literatura de autoayuda y psicología humanista, también de sitios web relacionados con estilos de vida saludables y bienestar espiritual. De hecho, sus menciones a estos temas en las juntas terapéuticas solían levantar ciertas suspicacias entre sus compañeros, acostumbrados como mucho a encontrarse con miembros que realizaban paralelismos entre sus experiencias en AA y en grupos religiosos, generalmente evangelistas. Al preguntar sobre sus proyectos de vida a futuro, ya sin el alcohol, ambos articularon respuestas que llamaron mi atención por salirse de los parámetros en los que se encuadraban las respuestas del resto de sus compañeros. Estas hacían referencia a sus deseos de hallar el equilibrio consigo mismos y con los demás a través de una vida sana y equilibrada. Además esto tenía poco que ver con el alcohol; sus narraciones se vinculaban con cuestiones como mantener una alimentación sana, esencialmente vegetariana, que no los intoxicase, y hacían también referencia a la búsqueda del bienestar personal en un sentido que entonces, y aún hoy, tenía para mí resonancias new age, pero que se deslizaban entre las afirmaciones y máximas prototípicas de la literatura y el programa $A A$ con su particular idea de sobriedad. Este término en los grupos de AA recoge toda una serie de significados psicológicos, sociales y espirituales de la persona, que en su caso se materializaba en la idea de «resultar atractivos a la sociedad», invirtiendo el estigma del alcoholismo, pero que a la vez parecía ir bastante más allá de lo habitual.

Desde mi punto de vista, la cuestión con P. y E. tenía que ver sobre todo con el impacto de estas influencias new age en sus relatos vitales en un doble sentido. Por un lado, las narrativas que estos miembros construían en tribuna ciertamente tendían a salirse de la pauta general del grupo. De alguna forma, en sus revisiones como miembros con experiencia en torno a las vivencias cotidianas o al significado del conjunto de su historial como alcohólicos era fácil que tanto uno como otro terminaran por ofrecer interpretaciones, consejos a sus compañeros, en las que aparecían ideas difusas sobre la energía, la predestinación y como cada persona atrae con su temperamento y el pasado de vínculos familiares determinado tipo de sucesos, que no terminaban de convencer a los compañeros más preocupados por vigilar una cierta ortodoxia para con el programa AA. $Y$ hay que señalar que esto no es algo baladí, esencialmente puede decirse que justo ahí radica la clave de la terapia $\mathrm{AA}$, más allá de dejar atrás su relación con la bebida; para aquellos que consiguen mantenerse sobrios en el programa las reuniones o juntas terapéuticas va más de reelaborar sus experiencias vitales diarias, los conflictos y emociones que enfrentan que de lidiar con la sustancia en sí. Por lo que el hecho de que la clave interpretativa y de elaboración de dichas narrativas en estos dos miembros estuviese tematizada dentro de una lógica extraña representaba para muchos un problema de cohesión, un peligro para el grupo, por más que en algunos aspectos ambos marcos pudieran encajar.

Por otra parte, algo similar sucedía con sus vidas cotidianas, o mejor dicho, con el ejemplo que representaban para sus compañeros. Tanto uno como otro formaban parte de la reducida pero activa red de personas de la ciudad interesadas en todo lo que tuviera reminiscencias del llamado ambiente holístico. De manera que a menudo participaban de reuniones, cursos de meditación, talleres de manejo energético, uso de piedras con propiedades esotéricas o de cocina hinduista. Esto era visto por muchos de sus compañeros como algo negativo que los desprestigiaba como ejemplos de sobriedad para los miembros con menos experiencia. El grueso de ese tejido de relaciones sociales, intercambio de información o actualización de las terapias o mecanismos de trabajo espiritual en boga, estaba constituido, según relataban, por mujeres de clase media alta de la ciudad, aunque también incluía a algunos hombres. En muchos casos dichas actividades constituían bajo la perspectiva de sus compañeros un obstáculo para que E. y P. participaran de las reuniones con ellos fuera de las juntas, y también para que estos decidieran implicarse en labores organizativas de la agrupación o de apadrinamiento de compañeros con menos experiencia.

Al fin y al cabo, teniendo en cuenta la capacidad de adaptación a diferentes contextos culturales que ha demostrado el programa de $A A$, la inserción de lógicas new age entre las creencias o marcos explicativos de sus miembros seguramente no represente un peligro para dicho modelo terapéutico. Ya en la actualidad pueden encontrarse sin salir de México agrupaciones de AA de lo más variado, algunas por ejemplo muy cercanas a movimientos religiosos protestantes u otras que solo cuentan entre sus miembros con profesionales de la medicina. Lo interesante de todo esto radica quizá en que nos informa de procesos de hibridación, de entrecruzamiento de las lógicas espirituales y terapéuticas de los llamados movimientos alternativos, con otras lógicas prove- 
nientes de ámbitos aparentemente alejados, pero con los que puede conectar y encontrar posibilidades fértiles de variación.

\section{TURISTAS RESIDENCIALES EUROPEOS EN EL SUDESTE PENINSULAR: LA BÚSQUEDA DE LA FELICIDAD AUTÉNTICA}

Como segundo segmento etnográfico para apoyarnos en la reflexión, partiremos de un fenómeno social que $a$ priori, quizás presente incluso una mayor distancia relativa con el cruce entre nuevas espiritualidades y prácticas alternativas en salud que el caso de los grupos de AA. Aunque como es el caso aquí, también es posible verlo como un ámbito en principio bastante lejano, en el que de nuevo apreciar la reverberación de estas lógicas da pie para pensar sobre las posibilidades de encaje que este tipo de prácticas guardan con estilos de vida concretos, en los que la búsqueda de marcos de sentido emancipatorios ocupa un espacio central y cobran un sentido cualitativamente diferente.

Este segmento parte de las impresiones surgidas a propósito de una investigación socioantropológica centrada en el fenómeno del turismo residencial europeo de interior. Un fenómeno que tuvo su mayor auge hace algunos años, pero que a pesar de no recibir tanta atención por parte de los medios de comunicación o las ciencias sociales aún perdura. Dicho turismo residencial consistió en que una buena cantidad de ciudadanos europeos en edad de retiro (sobre todo británicos) optaron por establecer sus proyectos de vida de manera estable en zonas del interior del sureste de la península ibérica (Rodes 2011). La principal característica de este fenómeno radicaba en que, en principio, constituía un tipo de turismo y de turista alternativos a los que tradicionalmente han imperado en países como España, el conocido modelo de sol y playa.

Una buena parte de los turistas residenciales de interior con los que contactamos durante el trabajo partían de proyectos de vida bastante similares. Esencialmente se trataba de personas de clase media, en pareja, de profesiones liberales, que habían adquirido y en muchos casos participado activamente en la rehabilitación antiguos caseríos, casas abandonadas en parajes rurales e incluso casas cueva. Además, en las narraciones sobre estos proyectos, aparecían también ideas vinculadas con la búsqueda de experiencias de autodescubrimiento, de contacto con la realidad local, y cómo no, de transformación personal (Palacios, Jiménez y Rico 2013). Como parte del proyecto pudimos obtener, a partir de un cuestionario abierto, respuestas sobre la orientación de dichos proyectos de vida, coincidentes con los llamados «estilos de vida globales» (Collier y Lakoff 2004). Estas respuestas, se vinculaban usualmente con la búsqueda de la felicidad, del bienestar, vinculados aquí con el sol, el clima, la salud, y también con la huida de lo que llamaban «la trampa», refiriéndose a sus situaciones en sus países de origen. Y se manifestaba además en su participación en la elaboración de artesanías y la rehabilitación de huertos ecológicos y viviendas tradicionales.

Al no ser parte del foco de interés del planteamiento de la investigación, y también en parte debido a que en este caso la misma se desarrolló por parte de un equipo en el que pudimos contar con personas que realizaron buena parte del trabajo de campo, a diferencia del caso de $A A$, aquí no cuento con una base empírica convencional que me permita sostener aseveraciones rigurosas sobre la presencia en las vidas de los turistas residentes de elementos del ambiente holístico. Como señalaba al inicio de este apartado, tampoco es exactamente mi intención. Lo que sí pretendía señalar, y me parece que es una reflexión lo suficientemente sugerente como para que merezca la pena plantearla, es que ese marco de ideas que antes mencionaba, esas concepciones cuasi trascendentales en torno a la búsqueda de una felicidad auténtica, parecen guardar algún tipo de relación con las articulaciones de nuevas espiritualidades y prácticas alternativas en salud de las que venimos hablando. Es posible que no sea una relación directa observacionalmente demostrable en un sentido que permita hablar de una clara presencia de estas prácticas, tal y como se delimitan en la literatura teórica, entre estos grupos de población. Pero sí que me parece que desde su mismo origen dichas ideas de bienestar y autenticidad están marcadas por este espíritu, el espíritu de una época en la que predominan el llamado a que las personas desarrollen con sus vidas esos ejercicios de búsqueda, desplazamiento y transformación personal, sea moviéndose en el espacio, sea moviéndose a través de marcos culturales, opciones, proyectos vitales o identidades.

De manera particular, en el marco de sus «nuevas vidas» algunas de estas personas trataran de «reencantar el mundo» mediante el sostenimiento de prác- 
ticas ligadas de forma más o menos clara al ambiente holístico o de otras no tan directamente relacionadas. Independientemente de ello, esa articulación trascendentalista de la búsqueda personal de nuevos marcos instituidos a partir de la experiencia, que en cierto modo casi sacraliza la estetización de la vida cotidiana, la actividad artesanal, el contacto con la naturaleza, puede considerarse uno de los sustratos esenciales de las filosofías y estéticas ambientalistas desde su inicio ${ }^{7}$, de la misma manera que la vinculación de la búsqueda de una nueva identidad externa a los marcos y convenciones socioculturales propios forma también parte del acervo de experiencias de las tradiciones contraculturales también desde sus orígenes.

Como decía, la temática y la dinámica de la investigación no permitieron en su momento que pudiera profundizar en estas impresiones. Sin embargo, con el paso del tiempo y el desarrollo del interés por estas cuestiones, no he dejado de encontrar pequeños indicios que no dejaban de reforzar dichas sensaciones. Así con posterioridad he tenido la oportunidad de conocer de primera mano, y también a través de noticias de conocidos comunes, casos de personas venidas de otros países europeos que cumplían el perfil de la citada investigación y que de algún modo sí que parecían reflejar esa conexión. Es el caso por ejemplo de $M$. una mujer de origen holandés instalada hace más de 10 años en una pequeña población de interior del sudeste, a medio camino entre la sierra y la zona de costa. Conocí a $\mathrm{M}$. a través de unas colegas del ámbito académico. Contaba con un nivel formativo medio, en su país de origen había estudiado Enfermería, además de desarrollar muy distintos trabajos como parte de los viajes que junto a su por entonces pareja la habían traído hasta España. En algunas de las charlas informales que pude sostener con ella, ya a posteriori de la citada investigación, sí que pude plantearle mis impresiones. Para nada constituía un caso de persona volcada en el tipo de prácticas holísticas que hemos venido citando, pero sí que hacía con asiduidad yoga, decía tratar de respetar los preceptos alimentarios de pureza/impureza ligados a dicho sistema y, de manera dispersa, mostraba interés en conversaciones y lecturas por dichas temáticas. Pero sobre todo, y eso es lo que hace que me parez-

7 Puede verse por ejemplo el reciente ensayo de Wulf (2016) sobre la influencia de las ideas de Alexander Von Humboldt en el ambientalismo. ca un caso reseñable entre otros similares que he ido encontrando con posterioridad y que guardan una relación más claramente directa con nuestro ámbito de reflexión, afirmaba encontrar un vínculo, una relación significativa entre lo que suponía la transformación fruto del viaje y del cambio de plan vital, con la "toma de conciencia», el cambio que también suponían a su juicio la asunción de posiciones alternativas en lo relativo al significado de las experiencias, el desarrollo del autoconocimiento y el cultivo de otras formas de pensar.

\section{EN CLAVE DE SÍNTESIS}

Pero qué pretendíamos hacer ver con estos fragmentos etnográficos y qué relación guardan con los puntos de anclaje teóricos que habíamos esbozado previamente en torno a las articulaciones entre los sistemas de prácticas alternativas en salud y nuevas espiritualidades. En primer lugar, que evidentemente resulta muy complicado determinar el grado de significatividad de la hibridación en las prácticas de elementos de la esfera holística con otros de ámbitos como el modelo AA o con tipos de experiencias ligadas a la búsqueda de un bienestar trascendentalizado, como el turismo residencial. Del mismo modo que en términos generales puede resultar complejo intentar estimar en términos cuantitativos el grado de extensión de las prácticas del llamado ambiente holístico, esto es, cuántas personas realizan prácticas de éste tipo. Incluso podría resultar divertido pensar en delimitar dentro de ciertas convenciones sociológicas los criterios para una supuesta estimación. Así, para averiguar qué grado de pureza se determinaría en las distintas prácticas, bastaría con una persona como sujeto de una práctica puntual, o qué sucedería con aquellos que sostienen muchas y variadas, cuando además éstas van cambiando. Y sobre todo, hasta qué punto la búsqueda de las manifestaciones que mejor encajan, en términos de significatividad y relevancia, con nuestros esquemas teórico-metodológicos, puede hacer que estemos dejando de lado toda una serie de manifestaciones que sencillamente pueden parecernos espurias o irrelevantes, pero que al fin y al cabo forman parte de los procesos de búsqueda de sentido de determinados grupos de personas, y que quizás podrían ser incluso mayoritarios estadísticamente hablando, dependiendo de los criterios que se pudiesen argumentar. 
En segundo lugar, y vinculado precisamente con la cuestión del hibridismo en las prácticas culturales (Burke 2010), la extensión mundial de sistemas de prácticas como los mismos AA o la búsqueda de la experiencia de reenmarque que supone trasladarse a vivir en el marco de otra cultura, son un claro ejemplo del grado de apertura que exhiben estas nuevas/ viejas articulaciones entre espiritualidad y formas de autocuidado. La clave de su extensión es su porosidad, su capacidad para ser adaptadas en muy diversos contextos locales. Y lo que es más interesante, parece que, en su diversidad de conformaciones, ofrecen sabidurías y tipos de experiencias que pueden permitir reconfigurar el sentido de las narrativas biográficas de quienes los sostienen. En tercer lugar, en lo relativo a los proyectos de vida de los turistas/ residentes de interior, no solo se trata de que estas prácticas muestren ejemplarmente la ubicuidad, el carácter difuso de esta especie de ondas expansivas, de relieves del ambiente holístico. Se trata de que las claves concretas de la articulación y la significación de estas prácticas, sus efectos se localizan en trayectorias vitales y contextos cotidianos de las personas que las sostienen, no tanto en los escenarios públicos y semiprivados en los cuales nuestras ópticas teórico metodológicas están preparadas para apreciar cosas.

\section{TODAS LAS POTENCIALES POSIBILIDADES POR VENIR (A MODO DE REFLEXIONES FINALES)}

\section{LOS DILEMAS DE TRATAR DE APREHENDER LO NUEVO}

No cabe duda que el despliegue en nuestras sociedades de formas de producción de experiencias, subjetividades e intersubjetividades (Biehl, Good y Kleinmann 2007) desborda prácticamente cualquier intento de sistematización de la cuestión. Las prácticas sociales que pueden considerarse inmersas en las ondas expansivas de la diseminación de las lógicas del ambiente holístico, ponen encima de la mesa una doble cuestión respecto a su contemporaneidad (Foucault 1991; Rabinow 2007: 2). Por un lado, el interés por los amplios procesos precedentes, que de algún modo serían los cauces que sirven de condiciones de posibilidad a estas nuevas formas de subjetividad. Por otro, las posibilidades a futuro, los perfiles de cambio que este tipo de prácticas parecen anunciar, aunque sea potencialmente ${ }^{8}$. Lo que parece claro es que dichas prácticas reflejan de forma clara la complejización del repertorio de conocimientos, concepciones, sistemas de prácticas y marcos éticos, desde y entre los cuales se están configurando las nuevas formas de objetivar y problematizar las experiencias. $Y$ en ese punto es donde parece que la cuestión se torna extremadamente compleja, ya que, al margen de constatar la presencia de los llamados discursos sociales, marcos éticos que parecen conducir o predisponer a los individuos a adoptar dichos marcos y responsabilizarse creativamente de sus vidas, movilizaría la cuestión de las influencias o efectos de los marcos macro de condiciones. Aparece como clave de valor insoslayable la cuestión del sentido, respecto en qué marcos, límites o convenciones se movilizan y articulan esas prácticas, y cómo son percibidas por quienes las sostienen, aunque sea de una manera inmediata, como formas de contestación, respecto de qué consideran que se están emancipando, qué libertad creen que están conquistando.

Aun partiendo de planteamientos comunes alrededor de la producción tecnosocial, el ensamblaje de los nuevos sujetos y subjetividades, las principales posiciones posestructuralistas respecto al abordaje de la historicidad de estas prácticas alternativas, en el sentido en que suponen la emergencia de algo nuevo, y el modo en que esto se produce pueden ofrecer matices interesantes. Si desde el punto de vista foucaultiano, y su interés por enfatizar el papel de las determinaciones sociohistóricas, se enfatiza en el cuestionamiento por las posibles alineaciones no percibidas desde la práctica entre las estrategias políticas y la búsqueda de formas de libertad (Foucault 2002b). Del lado del pensamiento de Gilles Deleuze (Deleuze y Guattari 2002) se enfatiza la segmentariedad entre los dominios macro y micro ${ }^{9}$, la virtualidad

8 Resulta curioso que la misma percepción de encontrarse ante una especie de cambio de época es operada a su manera por los propios actores sociales, ya que como nos recuerda en clave sociológica De Marinis (2010: 331), al preguntarse por la efervescencia de las formas comunitarias en las sociedades actuales, estas remiten a tres dimensiones espacio/tiempo con sus respectivas connotaciones morales: pasado y la nostalgia de la tradición; presente y la posibilidad; y el futuro y las utopías.

9 «Toda sociedad, pero también todo individuo, están, pues, atravesados por las dos segmentariedades a la vez: una molar y otra molecular. Si se distinguen es porque no tienen los mismos términos, ni las mismas relaciones, ni la misma naturaleza, ni el mismo tipo de multiplicidad. Y si son inseparables es porque coexisten, pasan la una a la 
de las revoluciones potenciales que encarnan las singularidades producto de las interrelaciones entre las búsquedas de individuos y grupos y su aprehensión por los sistemas ideológicos y culturales hegemónicos.

\section{LAS NUEVAS SUBJETIVIDADES Y EL PODER}

En ese sentido, tal y como señalan muchos autores (Cornejo, Blázquez y Flores 2014), es relativamente común que, desde los análisis sobre el ambiente holístico, surja la cuestión de en qué medida puede considerarse entonces como "la espiritualidad del neoliberalismo». Seguramente lo más apropiado antes de abordar esto, sería tratar de aclarar qué se está entendiendo en cada caso por neoliberalismo. El sociólogo Loïc Wacquant (2012) proponía en su momento una caracterización de las principales opciones, que lógicamente le servían para establecer la suya como contrapunto. De un lado, tendríamos análisis que parten de definir el neoliberalismo como un «sistema productivo» (Harvey 2009), ligado con la extracción y distribución de la riqueza y los recursos, que tendría toda una serie de efectos tecnológicos, sociales, espaciales, poblacionales, e incluso psicológicos (Sennet 2000), cuya matriz lógica sería la mercantilización de la vida y los recursos sociales. Del otro Wacquant sitúa aquellos puntos de vista que ve en el neoliberalismo una "racionalidad de gobierno", una tecnología política de organización de la vida en distintos sentidos (biológico, social, epistemológico), que ensambla diseños, problemas, intervenciones y sujetos a nivel global, que luego se aterrizan o ajustan a nivel local (Ong y Collier 2004).

Ciertamente las perspectivas que para explicar el neoliberalismo focalizan su atención en estructuras y procesos económico-políticos, ofrecen claves en las que encuadrar las ondas expansivas del ambiente holístico. En parte los nuevos sujetos, espirituales y terapéuticos, se articulan en múltiples modulaciones entre la feligresía y el consumo. Y es cierto que hay toda una serie de cuestiones jurídico-científicas que

otra (...). En resumen, todo es política, pero toda política es a la vez macropolítica y micropolítica» (Deleuze y Guattari 2002: 218). Desde otro registro bastante distinto, Norbert Elias (2011) diferenciaba dos dimensiones en el proceso civilizatorio: la que afectaba a estructuras sociales y políticas (sociogénesis) y la que afectaba a estructuras psicológicas y cognitivas (psicogénesis). cristalizan en torno a la discusión y regulación de la legitimidad de la emergente industria de las terapias alternativas, o las más o menos impostadas preocupaciones por los nuevos grupos religioso-espirituales y la ocasional aparición de sectas. Pero la complejidad de las posibilidades e implicaciones de estas prácticas escapa con mucho a estos esquemas. El sentido de ideas como el bienestar, la búsqueda espiritual o la prevención de las enfermedades desde las prácticas cotidianas dependen en realidad de constelaciones cuasi ideológicas concretas y particulares, relacionalmente entendidas con sus posiciones y oposiciones dentro de marcos sociopolíticos locales concretos. Pero sobre todo guarda relación con trayectorias y situaciones individuales y colectivas particulares respecto a las cuales representan el repertorio predominantemente disponible de respuestas.

Realmente me parece que el dilema sobre calificar como neoliberales o no a las prácticas vinculadas con el ambiente holístico no está relacionado exactamente con dirimir su ideología. Mi impresión es que de manera inevitable, ante este tipo de sistemas de prácticas a menudo nos preguntamos dónde está el límite entre la coerción persuasiva y el empoderamiento liberador ${ }^{10}$, de cara a caracterizar el sentido de la acción de los sujetos individuales o colectivos que observamos.

En una conocida entrevista realizada a comienzos de los años ochenta, Foucault (2001: 245-246) hablaba de «la importancia en nuestros días de las luchas contra las formas de sujeción, contra lo que liga al individuo a sí mismo y lo somete a sí mismo», para después introducir en la argumentación la importancia de un tipo de tecnología del poder que denominaba "pastoral», y que vinculaba con el cristianismo de la antigüedad, definiéndolo como una forma de poder cuyo objetivo sería «conseguir la salvación individual en el más allá». Estas técnicas pastorales

10 A partir de la reorientación de su teoría del poder, con el abandono de una concepción restrictiva y extractiva en pos de una productiva y regulatoria, la cuestión del sujeto y la subjetividad, se torna problemática en el trabajo de Foucault (2002b), debido a la articulación de formas de subjetivación coactivas, externas y heteronómicas, autónomas y liberadoras, que permiten a los sujetos constituirse en sujetos éticos: "Hay dos significados de la palabra sujeto: por un lado, sujeto a alguien por medio del control y de la dependencia, por otro, ligado a su propia identidad por conciencia y autoconocimiento. Ambos significados sugieren una forma de poder que subyace y sujeta» (Foucault 2001: 245). 
habrían sido aprehendidas, resignificadas y transformadas posteriormente en el marco de las formas modernas de gobierno. Cabe pensar que aunque estas nuevas pastorales contemporáneas pueden incluir distintos conjuntos de prácticas ligados a otros campos sociales (véase viejos y nuevos populismos, comunicación de masas, neotradicionalismos...), no parece extraño que su centralidad en las racionalidades políticas modernas, sea el punto de conexión, de actualización de las antiguas pastorales en nuevas constelaciones postliberales de sentido, donde ahora lo sacralizado son la identidad personal, el yo, junto a determinadas ideas de libertad, autonomía o bienestar ${ }^{11}$. Así también es posible que los viejos marcos sobre la ideología, las creencias o los cambios sociales que a menudo ponemos en juego sin ser totalmente conscientes no resulten válidos para aprehender lo que pueden suponer este tipo de fenómenos.

\section{LAS POSIBILIDADES POR VENIR}

Del mismo modo que resulta complejo interpretar hasta qué punto sostener un determinado tipo de prácticas libera o emancipa a una persona respecto a determinadas circunstancias y/o condiciones, resulta igualmente difícil captar los momentos en que esos descontentos y malestares se articulan de un modo colectivo, en algún tipo acción social de cambio, o que dichos cambios alcanzan o no cierto grado de extensión social. Así, los conceptos de corte deleuziano parecen señalar otras vías de análisis, que, por ahora, se han centrado en movimientos sociales emergentes (Fernández de Rota 2008). Y en algún caso concreto, como el del antropólogo João Biehl, el aparataje conceptual deleuziano es utilizado para aproximarse a las trayectorias vitales e institucionales de personas en situaciones límite de exclusión y con trastornos clínicos de difícil manejo ${ }^{12}$ (puede verse también Guattari 2005).

11 «Ya no se trata de conducir a la gente hacia la salvación en el más allá, sino más bien de asegurársela en este mundo. $Y$ en este contexto, la palabra salvación adquiere un significado diferente: salud, bienestar (esto es riqueza suficiente, buen nivel de vida), seguridad, protección contra los accidentes» (Foucault 2001: 247).

12 «Through close attention to people moving through broken institutions and infrastructures in the making and with careful observation always complicating the a priori assumptions of universalizing theory, ethnographic work can make public the constellations through which life chances
Tratar de visualizar las potenciales vías de mutación del ambiente holístico resulta tan apasionante como complicado, pues parecen poder abarcar una tensión que iría desde articularse con formas de vida alternativas, digamos que con un pie dentro y otro fuera del sistema, hasta derivaciones publicitarias donde parece encajar perfectamente con una especie de nuevo consumismo cognitivo (decoración, cocina, belleza). De manera general, se han extendido y vulgarizado determinados principios o ideas básicas del ambiente holístico fuera de sus límites y comunidades de sentido, ideas como la búsqueda de la felicidad, el bienestar o la energía siendo integradas y utilizadas como explicaciones con poco o nulo contacto con el ambiente holístico, incluso integrándose con programas terapéuticos o movimientos religiosos de distinto origen, pero también en la conjunción de nuevas formas de buena vida, en este caso globalizadas. Igualmente resulta seductora la idea de no perder de vista los procesos de interacción, contestación, pero también de integración y traducción entre los principios del ambiente holístico y las nuevas formas de subjetividad producidas y extendidas desde ámbitos no alternativos. Todo ello, al fin y al cabo, contemplado desde la óptica que venimos caracterizando, no significa para nuestra tarea como antropólogos, más que el reto de vislumbrar tendencias sobre las cuales pensar, pero también con las cuales comprometerse o establecer vías de crítica.

\section{BIBLIOGRAFÍA CITADA}

Antze, Paul. 1987. "Symbolic Action in Alcoholics Anonymous», en Mary Douglas (ed.), Constructive Drinking: Perspectives on Drink from Anthropology: 149-181. Nueva York: Cambridge University Press.

Baer, Hans A. y Robbie Davis-Floyd. 2006. «Health Care, Alternative», en James H. Birx (ed.), Encyclopedia of Anthropology: 1146-1148. Thousand Oaks CA: Sage Publications.

\footnotetext{
are foreclosed and highlight the ways desires can break open alternative pathways. [...] The question, rather, lies in our receptivity to others, in what kinds of evidence we assemble and use-the voices to which we listen and the experiences we account for-and in how we craft our explanations: whether our analytics remain attuned to the intricacy, openness, and unpredictability of individual and collective lives. Just as medical know-how, international political dynamics, and social realities change, so too are people's lives (biological and political) in flux» (Biehl y Locke 2010: 318).
} 
Beck, Ulrich, Anthony Giddens y Scott Lash (eds.). 1997. Modernización reflexiva. Política, tradición y estética en el orden social moderno. Madrid: Alianza.

Biehl, João, Byron Good y Arthur Kleinman. 2009. «Introduction: Rethinking Subjectivity», en Joao Biehl, Byron Good y Arthur Kleinman (eds.), Subjectivity Ethnographic Investigations: 1-23. Los Angeles: University of California Press.

Biehl, João y Peter Locke. 2010. «Deleuze and the Anthropology of the Becoming». Current Anthropology 51(3): 317351.

Biehl, João y Amy Moran-Thomas. 2009. «Symptom: Subjectivities, Social Ills, Technologies». Annual Review of Anthropology 38: 267-288.

Blázquez Rodríguez, Maribel y Mónica Cornejo Valle. 2013. «Empoderamiento de género en las medicinas alternativas y complementarias (MAC) de influencia new age ¿es el holismo feminista?», en Heriberto Cairo y Lucila Finkel (coord.), Actas XI Congreso Español de Sociología: 1377-1385. Madrid: Federación española de Sociología/Universidad Complutense. Disponible en: <http://www.fes-sociologia.com/ files/congress/11/Libro\%20de\%20Actas\%20final_1.pdf>. Fecha de acceso: 20 may. 2014

Blázquez Rodríguez, Maribel y María Jesús Montes. 2010. "Emociones ante la maternidad: de los modelos impuestos a las contestaciones de las mujeres». Ankulegi 14: 81-92.

Bourdieu, Pierre. 1997. Razones prácticas. Sobre la teoría de la acción. Barcelona: Anagrama.

Bowman, Marion y Ulo Valk (eds.). 2012. Vernacular Religion in Everyday life: Expressions of Belief. Sheffield: Equinox.

Burke, Peter. 2010. Hibridismo cultural. Madrid: Akal.

Cain, Carole. 1991. «Personal Stories: Identity Acquisition and Self-Understanding in Alcoholics Anonymous». Ethos 19(2): 210-253.

Collier, Stephen J. y Andrew Lakoff. 2004. «On Regimes of Living», en Aiwa Ong y Stephen Collier (eds.), Global Assemblages: Technology, Politics, and Ethics as Anthropological Problems: 22-39. Nueva York: Blackwell.

Collier, Stephen y Aihwa Ong. 2004. «Global Assemblages, Anthropological Problems», en Aiwa Ong y Stephen Collier (eds.), Global Assemblages: Technology, Politics, and Ethics as Anthropological Problems: 3-21. Nueva York: Blackwell.

Comelles, Josep M. 2003. «El regreso de lo cultural. Diversidad cultural y práctica médica en el s. XX|». Cuadernos de Psiquiatría Comunitaria 3(1): 7-21.

Comelles, Josep M. y Ángel Martínez Hernáez. 1993. Enfermedad, Cultura y Sociedad: un ensayo sobre las relaciones entre la Antropología Social y la Medicina. Madrid: Eudema.

Cornejo Valle, Mónica y Maribel Blázquez Rodríguez. 2013. «La convergencia de salud y espiritualidad en la sociedad postsecular. Las terapias alternativas y la constitución del ambiente holístico». Revista de Antropología Experimental 13: 11-30. Disponible en: < https://revistaselectronicas. ujaen.es/index.php/rae/article/view/1813/1569>. Fecha de acceso: 5 abr. 2014.
Cornejo Valle, Mónica, Maribel Blázquez Rodríguez y Juan A. Flores Martos. 2014. «El incómodo vínculo entre medicina y fe», en Mónica Cornejo Valle, Maribel Blázquez Rodríguez y Juan A. Flores Martos (comps.), El reencuentro de salud y espiritualidad: agencias, saberes y prácticas. Actas del XIII Congreso Nacional de Antropología. Periferias, fronteras y diálogos: 4485-4492. Tarragona: Universitat Rovira i Virgili.

De la Torre, Renee y Cristina Gutiérrez Zuñiga. 2005. «La lógica del mercado y la lógica de la creencia en la creación de mercancías simbólicas». Desacatos 18: 53-70.

De Marinis, Pablo. 2010. «Sociología clásica y comunidad: entre la nostalgia y la utopía (un recorrido por algunos textos de Ferdinand Toennies)», en Pablo De Marinis, Gabriel Gatti e Ignacio Irzuzta (eds.), La comunidad como pretexto. En torno al resurgimiento de las solidaridades comunitarias: 347-382. Barcelona: Anthropos.

Del Vecchio Good, Mary-Jo. 2007. «The Medical Imaginary and the Biotechnical Embrace: Subjective Experiences of Clinical Scientists and Patients», en Joao Biehl, Byron Good y Arthur Kleinman (eds.), Subjectivity Ethnographic Investigations: 362-380. Los Angeles: University of California Press.

Deleuze, Gilles y Félix Guattari. 2002. Mil mesetas. Capitalismo y esquizofrenia. Valencia: Pre-Textos.

Echevarría Pérez, Paloma. 2008. «Análisis antropológico de los patrones de uso y el perfil del usuario de las terapias complementarias orientales». Gaceta de Antropología 24(2), art. 27. Disponible en: <http://hdl.handle.net/10481/6917>.

Elias, Norbert. 2011. El proceso de la civilización: Investigaciones sociogenéticas y psicogenéticas. México DF: Fondo de Cultura Económica.

Esposito, Antonio. 2012. El dispositivo de la persona. Madrid: Amorrortu.

Fassin, Didier. 2008. Faire de la santé publique. París: Editions de L'École des Hautes Etudes.

Fedele, Anna. 2008. El camino de María Magdalena. Un recorrido antropológico por la ruta del peregrinaje de la nueva espiritualidad. Barcelona: Integral.

Fedele, Anna y Ruy Llera Blanes (orgs.). 2011. Encounters of Body and Soul in Contemporary Religious Practices: Anthropological Reflections. Nueva York: Berghahn Books.

Flores Martos, Juan A. 2011. «Interculturalidad en salud y eficacia: Algunas indicaciones de uso para OGND con proyectos de salud en América Latina». Nuevo Mundo, Mundos Nuevos, vol. 11. doi: <https://doi.org/10.4000/nuevomundo.61232>. Fecha de acceso: 25 feb. 2014.

Fernández de Rota, Antón. 2008. «Movimientos sociales. Una lectura a partir del posestructuralismo». Athenea Digital 14: 63-81.

Foucault, Michel. 1976. "Crisis de la medicina o de la antimedicina». Educación médica y salud 10(2): 152-170.

Foucault, Michel. 1991. "¿Qué es la Ilustración?», en Michel Foucault, Saber y Verdad: 197-207. Madrid: Las Ediciones de la Piqueta. 
Foucault, Michel. 1992. "Historia de la medicalización», en Michel Foucault, La vida de los hombres infames: 54-68. Buenos Aires: Altamira.

Foucault, Michel. 2001. «El sujeto y el poder», en Hubert Dreyfus y Paul Rabinow, Michel Foucault: Más allá del estructuralismo y la hermenéutica: 241-259. Buenos Aires: Nueva Visión.

Foucault, Michel. 2002a. La voluntad de saber. México DF: Siglo XXI.

Foucault, Michel. 2002b. La inquietud de sí. México DF: Siglo $\mathrm{XXI}$.

Giobellina Brumana, Fernando y Elda González Martínez. 2000. Umbanda. El poder del margen. Un estudio sobre religiosidad popular y experiencia social. Cádiz: Universidad de Cádiz.

Hacking, lan. 2001. ¿La construcción social de qué? Barcelona: Paidós.

Han, Byung-Chul. 2014. Psicopolítica. Neoliberalismo y nuevas técnicas de poder. Barcelona: Herder.

Haro Encinas, Jesús A. 2000. «Cuidados profanos una dimensión ambigua en la atención de la salud», en Enrique Perdiguero y Josep M. Comelles (eds.), Medicina y cultura. Estudios entre la Antropología y la Medicina: 101-162. Barcelona: Bellaterra.

Harvey, David. 2009. Breve historia del neoliberalismo. Madrid: Akal.

Heelas Paul y Linda Woodhead. 2008. The Spiritual Revolution. Why religion is giving way to spirituality. Oxford: Blackwell.

Hervieu-Léger, Daniele. 2005. «Bricolage vaut-il dissémination? Quelques réflexions sur l'opérationnalité sociologique d'une métaphore problématique». Social Compass 52: 295-308.

Inhorn, Marcia. 2006. «Defining Women's Health: A Dozen Messages from More Than 150 Ethnographies». Medical Anthropology Quaterly 20(3): 345-378.

Janzen, John M. 2001. «Mind/Body, Subject/Object: Recent Trends in Medical Anthropology». Reviews in Anthropology 30: 343-360.

Lowenberg James y Fred S. Davis. 1994. «Beyond medicalisation-demedicalisation: the case of holistic health". Sociology of Health \& IIIness 16(5): 579-599.

Massó Guijarro, Esther. 2015. «Una etnografía lactivista: la dignidad lactante a través de deseos y políticas». Revista de Antropología Iberoamericana 10(2): 231-257.

Menéndez, Eduardo L. 1978. «El modelo médico y la salud de los trabajadores», en Franco Basaglia, Elio Giovannini, Silvano Miniati et al., La salud de los trabajadores. Aportes para una política de la salud: 11-53. México: Nueva Imagen.

Menéndez, Eduardo L. 2009. «De rituales y subjetividades. Reflexiones sobre algunas características de los grupos de Alcohólicos Anónimos». Desacatos 29: 107-120.

Menéndez, Eduardo L. y Renee Di Pardo. 1982. Alcoholismo: características y funciones del proceso de alcoholización; alienación, enfermedad o cuestionamiento. México DF: CIESAS.

Moreno Pestaña, José L. 2016. La cara oscura del capital erótico: capitalización del cuerpo y trastornos alimentarios. Madrid: Akal.

Ong, Aihwa. 2006. «Experiments with Freedom: Milieus of the Human». American Literary History 18(2): 229-244.

Ong, Aiwa y Stephen Collier (eds.). 2004. Global Assemblages: Technology, Politics, and Ethics as Anthropological Problems. Nueva York: Blackwell.

Palacios Ramírez, José. 2009. «La construcción del alcohólico en recuperación. Reflexiones a partir del estudio de una comunidad de Alcohólicos Anónimos en el norte de México». Desacatos 29: 47-68.

Palacios Ramírez, José, Modesto Jiménez García y Juan I. Rico Becerra. 2013. «Problematizando el turismo residencial europeo: tecnologías globales de gobierno y formas de subjetivación». Tabula Rasa 19: 167-189.

Palacios Ramírez, José y Juan I. Rico Becerra. 2011. «Globalización, Salud y Cultura: aspectos emergentes. Propuestas para el análisis desde la Antropología Social». Saude e Sociedade 20(2): 273-286.

Quiceno, Japcy M. y Stefano Vinaccia. 2009. «La salud en el marco de la psicología de la religión y la espiritualidad». Revista Diversitas. Perspectivas en Psicología 5(2): 321-336.

Rabinow, Paul. 1988. "Beyond ethnography: Anthropology as nominalism», Cultural Anthropology 3(4): 355-364.

Rabinow, Paul. 2007. Marking Time: On the Anthropology of the Contemporary. Princeton: Princeton University Press.

Rabinow, Paul y Nikolas Rose (eds.). 2003. The essential Foucault: selections from the essential works of Foucault. 19541984. Nueva York: New Press. Disponible en: <http://www2. Ise.ac.uk/sociology/pdf/RabinowandRoselntrotoEssentialFoucault2003.pdf>. Fecha de acceso: 12 oct. 2016.

Rodes, Joaquín. 2011. Residir aquí y allí. Estudio socio-antropológico de la movilidad europea. Murcia: Ediciones Isabor.

Rose, Nikolas. 1997. «El gobierno en las democracias liberales "avanzadas": del liberalismo al Neoliberalismo». Archipiélago. Revista de Pensamiento crítico 29: 25-40.

Rose, Nikolas. 1998. Inventing ourselves. Psychology, Power and Personhood. Cambridge: Cambridge University Press.

Rose, Nikolas. 1999. Powers of Freedom. Reframing Political Thought. Cambridge: Cambridge University Press.

Rose, Nikolas. 2003. "Identidad, genealogía e historia», in Stuart Hall y Paul Du Gay (comps.), Cuestiones de identidad cultural: 214-250. Buenos Aires: Amorrortu.

Rose, Nikolas. 2006. The Politics of Life Itself: Biomedicine, Power, and Subjectivity in the Twenty-First Century. Princeton: Princeton University Press.

Scheper-Hughes, Nancy y Margaret Lock. 1987. «The Mindful Body: A Prolegomenon to Future Work in Medical Anthropology». Medical Anthropology Quarterly 1(1): 6-41. 
Valverde, Mariana. 1998. Diseases of the Will. Alcohol and the Dilemas of Freedom. Nueva York: Cambridge University Press.

Vázquez, Francisco. 2005. Tras la autoestima. Variaciones del yo expresivo en la modernidad tardía. San Sebastián: Gakoa.

Wacquant, Loïc. 2012. "Three Steps to a Historical Anthropology of Actually Existing Neoliberalism». Social Anthropology 20(1): 66-79.
Weidner-Maluf, Sonia. 2005. «Mitos coletivos, narrativas pessoais: cura ritual, trabalho terapêutico e emergência do sujeito nas culturas da nova era». Mana. Estudos de Antropologia Social 11(2): 499-528.

Wulf, Andrea. 2016. La invención de la naturaleza. El mundo nuevo de Alexander V. Humboldt. Madrid: Taurus. 
